\title{
Modernización de autómatas programables, un análisis en la selección primaria
}

\author{
Bermúdez Carlos ${ }^{1}$ \\ ORCID: https://orcid.org/ 0000-0002-8763- \\ 5513 \\ Carlos.bermudez@gmail.com \\ UNEXPO, Vice Rectorado Puerto Ordaz \\ Estado Bolívar, Venezuela.
}

\author{
Ceballos Bejarano Edison Wernher ${ }^{2}$ \\ ORCID: https://orcid.org/ 0000-0002-6830- \\ 0355 \\ eceballos@unsa.edu.pe \\ Universidad Nacional de San Agustín de \\ Arequipa, Perú
}

\author{
Ceballos Bejarano Ferdinand Eddington ${ }^{3}$ \\ ORCID: https://orcid.org/0000-0003-2867- \\ 2397 \\ fceballos@unsa.edu.pe \\ Universidad Nacional de San Agustín de \\ Arequipa, Perú
}

Recibido (09/11/20), Aceptado (18/11/20)

\begin{abstract}
Resumen: Se presenta el análisis de un trabajo de investigación desarrollado en la empresa C.V.G CARBONORCA de Venezuela, la cual cuenta con dos plantas depuradoras de gases para el área de cocción, destinadas a purificar el gas que proviene de los hornos de cocción. Cada planta está conformada por electroválvulas, válvulas neumáticas, trasmisores, panel mímico del proceso y un sistema supervisorio. Todos estos elementos son gobernados por un PLC SIEMENS S5-115U el cual se encuentra en estado de obsolescencia, razón por la cual se diseñó la sustitución de estos autómatas por autómatas ContolLogix de ALLEN BRADLEY,con el fin de garantizar la continuidad en las operaciones en la planta. La investigación se hizo con un diseño descriptivo del tipo experimental de campo. Se obtuvo un código para cada planta depuradora de gas en RSLOGIX 5000 v17.00.00 y la actualización de la base de datos del sistema supervisorio. También se comprobó el funcionamiento del programa a través de una simulación de la planta en un sistema supervisorio, cuyo despliegue se diseñó para ese fin
\end{abstract}

Palabra Claves: Autómata, Modernización, ControlLogix, Sistema Supervisorio, Panel Mímico.

\section{Modernization of programmable automatons, an analysis in the primary selection}

\begin{abstract}
The analysis of a research work developed in the company C.V.G CARBONORCA of Venezuela is presented, which has two gas purification plants for the cooking area, designed to purify the gas that comes from the cooking ovens. Each plant is made up of solenoid valves, pneumatic valves, transmitters, process mimic panel and a supervisory system. All these elements are governed by a SIEMENS S5-115U PLC which is in a state of obsolescence, which is why the replacement of these automata by ALLEN BRADLEY ContolLogix automata was designed, in order to guarantee continuity in operations in plant. The research was done with a descriptive design of the field experimental type. A code for each gas treatment plant was obtained in RSLOGIX 5000 v17.00.00 and the update of the database of the supervisory system. The operation of the program was also verified through a simulation of the plant in a supervisory system, the deployment of which was designed for this purpose.
\end{abstract}

Keywords: Automation, Modernization, ControlLogix, Supervisory System, Mimic Panel

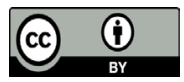




\section{I.INTRODUCCIÓN}

La empresa C.V.G. Carbones del Orinoco c.a. (CARBONORCA) es una empresa creada con el objetivo principal de producir ánodos de carbón, requeridos principalmente en los procesos de producción de aluminio en las empresas C.V.G. ALCASA y C.V.G. VENALUM. El proceso industrial de CARBORNORCA está dividido en dos módulos, el módulo de molienda y compactación y el módulo de hornos de cocción. En el módulo de hornos de cocción, se encuentra las plantas depuradoras de gases, las cuales permiten purificar los gases tóxicos que se generan en el proceso de cocción.

El sistema de control de las plantas depuradora de gases es gobernado por un PLC Siemnes S5-115U. Dicho PLC se encuentra desgastado, en vías de deterioro y ha sido descontinuado por el fabricante, por lo que requiere ser reemplazado. Asimismo, el departamento de Control de Procesos de C.V.G. CARBONORCA se ha propuesto como objetivo modernizar y estandarizar todos los autómatas programables de la empresa, que se encuentren en estado de obsolescencia.

En este trabajo se analiza el diseño de un trabajo de investigación que consiste en la modernización del autómata programable de las plantas depuradoras de gases, de la empresa C.V.G. CARBONORCA.

Estas plantas de depuración de gas están conformadas por equipos eléctricos, mecánicos, neumáticos y dispositivos electrónicos conformando un sistema de control gobernado por PLC's modelo S5 115U marca SIEMENS. Cada planta depuradora de gas es independiente de la otra, lo que implica que los sistemas de control son independientes entre sí.

El sistema de control incluye un panel mímico para cada planta, ubicado en la cabina de control y un sistema supervisorio basado en iFix Dynamics, el cual se comunica con el PLC a través de una interfaz RS232. En dicho sistema supervisorio es posible visualizar el despliegue del proceso, y enviar comandos a los PLC. Tanto en el panel mímico como en el sistema supervisorio, es posible visualizar los estados de las válvulas, los estados de las bombas, el nivel de los tanques asociados al proceso entre otras variables.

El trabajo está compuesto por cuatro secciones, en la segunda se plantean los factores teóricos de la investigación analizada, luego en la sección tres se evalúa la metodología y en la cuarta sección se exponen los resultados, finalmente se describen las conclusiones.

\section{DESARROLLO}

El sistema supervisor de la planta de aire comprimido de la empresa CVG Carabonorca fue optimizado en el año 2005 [1] se revisaron y depuraron las bases de datos del sistema supervisor iFIX (SISCO_S1 y SISCO_S2). Esto sirvió de guía para la actualización de la base de datos de las plantas depuradoras de gases LURGI 1.1 y LURGI 1.2. De manera similar la problemática planteada se tomó en cuenta con el fin de evitar la futura depuración de la base de datos a raíz de la modernización.

La base fundamental de la investigación analizada consistió en realizar la comunicación entre un autómata de la familia ControlLogix y el sistema supervisor con plataforma IFIX Dynamics. Para ello se trabaja con la base de datos denominada HDC_S3, la cual contiene todas las variables pertenecientes a la planta de hornos, dentro de las cuales se encuentran las plantas depuradoras de gases.

Otros autores [2] desarrollaron el sistema de control y supervisión para el sistema de manejo de ánodos cocidos (Cadena U) de CVG CARBONORCA. La cadena U es una línea de producción de la empresa la cual consiste en la extracción de los ánodos cocidos de los hornos y el apilamiento de dichos ánodos en un galpón destinado para este fin. Los ánodos son manipulados a través de una grúa puente. La cadena U es modernizada de un autómata de la marca MODICOM a un autómata de la marca Allen Bradley.

Más adelante, Pérez [3] realizó la modernización del sistema de control y supervisión local del manejo de ánodos verdes en la nave de cocción 1.2 de C.V.G. CARBONORCA. El objetivo del área de ánodos verdes es manipular y organizar los ánodos antes de entrar en el proceso de cocción. El autómata instalado en dicha área se encontraba obsoleto. El trabajo se basó en los PLC ControlLogix de AllenBradley e implementando el Panel View como interfaz hombre-máquina para la supervisión y el control local del sistema. Antiguamente esa área era gobernada por un autómata de la marca MODICOM.A diferencia del proyecto realizado por Ángel Pérez, el presente proyecto tiene como fin la modernización de la planta depuradora de gases de C.V.G. CARBONORCA y la migración de una plataforma SIEMENS a una plataforma ControlLogix. No se contempla el empleo de un panel View, y la arquitectura del hardware estará basada en expasión de Rack mediante redes ControlNet.

Mora [4] implementó el driver de comunicación entre el PLC S5-115U SIEMENS y el Sistema de supervi- 
sión iFIX Dynamics para supervisar el sistema de depuración de gases LURGI 1.2 de la empresa C.V.G CARBONORCA, planta que anteriormente no contaba con un sistema supervisor moderno. La metodología desarrollada permitió el rápido conocimiento y familiarización con las plantas depuradoras de gases y el sistema supervisorio de las mismas. Permitió también comprender la comunicación entre el autómata SIEMENS y el sistema supervisorio, información que fue vital para la comprensión del código grabado en el PLC. En lugar de diseñar una comunicación entre SIEMENS y iFix Dynamics, el presente proyecto diseña una comunicación entre Allen Bradley y iFix Dynamics.

Otras investigaciones [5] consistieron en el diseño del sistema de control para la bomba de alquitrán líquido del sistema de dosificación de la planta de molienda y compactación de C.V.G. Carbonorca. La problemática del proyecto se deriva de las constantes paradas que realiza la planta a raíz del mantenimiento que demanda la bomba que suministra alquitrán líquido y que es controlada por un módulo de control inteligente. Se diseña un despliegue para el sistema supervisorio basado en iFix, el mismo sistema que emplea el presente proyecto. Las experiencias de Rodriguez servirán de apoyo para la actualización de la base de datos las plantas depuradoras de gases así como para la elaboración de un despliegue para la simulación de la planta.

En este trabajo se analiza la modernización del autómata de la planta depuradora de gases, el cual es de la marca SIEMENS y se sustituiyó por un autómata más actual de la marca Allen Bradley. Se implementa a través de la plataforma ControlLogix.

\section{III.METODOLOGÍA}

Para este trabajo se consideró la siguiente población y muestra:

\section{A.Población}

La población constituye un conjunto de individuos o elementos que se presentan como partes del objeto de estudio. En este caso la población está representada por los autómatas existentes en las plantas depuradoras de gases LURGI 1.1 y LURGI 1.2.

\section{B.Muestra}

La muestra es un subconjunto representativo de un universo o población [6], en la metodología científica no es posible realizar investigaciones sobre la totalidad del fenómeno que interesa. En ese sentido, para efectos de este estudio, se define que la muestra poblacional son los autómatas Siemens S5 115U.

\section{C.Técnicas e instrumentos de recolección de datos}

En éste punto se describirá los instrumentos de recolección de información utilizados para llevar a cabo la elaboración de este proyecto.

-PLC ALLEN BRADLEY con procesador Logix5555, módulos de I/O y modulo de comunicación ControlNet.

-Cable serial RS232.

- Cable ControlNet.

- Cable para la comunicación con el PLC siemens S5

D.Programas de computadoras (Software)

-RSNetworx, software para la configuración de la red controlnet.

-RSLink, software que permite configurar y supervisar la(s) red(es) de comunicación(es) en la(s) que se encuentra conectado el autómata ControlLogix.

-RSLogix5000 v11.11, software que permite configurar, programar y supervisar el funcionamiento del autómata ControlLogix que tengan procesadores de la serie $55 \mathrm{xx}$

-RSLogix 5000 v 17, software que permite configurar, programar y supervisar el funcionamiento del autómata ControlLogix que tengan procesadores de la serie 56xx

-iFix Dynamics v2.5 y v3.0, software empleado para el sistema supervisorio

- Herramienta de configuración del driver de comunicación OPC, software para configurar el controlador de código abierto, empleado para comunicar el PLC con el sistema iFix Dynamics 
-Simatic Step5 version 7.2-21/11/01 para Windows XP, software para la programación, configuración y comunicación con el autómata Siemens de la serie S5

-Integrated Architecture Builder 4.02.03

\section{IV.RESULTADOS}

\section{A.Selección del PLC más apropiado para la modernización}

A continuación se listan las marcas y modelos de autómatas utilizados, equivalentes a un SIMATIC S5 y con presencia comercial.

Tabla 1. Marcas y modelos de autómatas

\begin{tabular}{|l|l|}
\hline Marca & Serie \\
\hline SIEMENS & SimaticS7 \\
\hline ALLENBRADLEY & ControlLogix \\
\hline Modicon & Quantum \\
\hline
\end{tabular}

Con cualquiera de estos PLC se puede implementar cualquier automatismo. Sin embargo cada marca ofrece facilidades para ciertas aplicaciones. Los costos de estos equipos varían levemente. Algunas marcas tienen mayor penetración comercial que otras y son capaces de ofrecer un mejor soporte técnico y mayor cantidad de repuestos. La selección del PLC apropiado se basó en el siguiente criterio:

-Innovaciones técnicas que ofrece cada autómata

-Estándares de diseño de la superintendencia de Control y procesos de C.V.G. Carbonorca

-Experiencias comerciales de C.V.G. Carbonorca con cada una de éstas empresas o distribuidores asociados a ellas

-Experiencias por parte del personal de C.V.G. Carbonorca con estos autómatas.

La superintendencia de control de procesos tiene el siguiente criterio de diseño para los nuevos automatismos a implementar dentro de la empresa:

\section{B. Estandarizar autómatas y protocolos de comunicación}

Este criterio consiste en trabajar con una sola marca de PLC en todas las plantas de la empresa. Inicialmente cada planta de la empresa fue diseñada con diferentes marcas de PLC, lo que obligó a la empresa a:

-Manejar una amplia gama de protocolos de comunicación que no siempre ofrecen compatibilidad entre sí

-Poseer un stock de repuestos muy amplio

-Adquirir una gran diversidad de licencias para paquetes de software para las diferentes marcas de autómatas presentes en la empresa

-Implementar numerosos protocolos de comunicación, lo que ha llevado a la adquisición de equipos especiales o adaptadores para crear la compatibilidad entre protocolos

$\mathrm{Al}$ estandarizar los autómatas con los que se trabajara en la empresa le da respuesta a los problemas técnicos derivados de la diversidad de protocolos de comunicación así como también se reducen costos ya que no será necesario poseer repuestos de diferentes proveedores y tampoco será necesario la adquisición de licencias de software para cada marca de PLC.

El estándar que ha adoptado la empresa desde hace 7 años es el siguiente:

-El protocolo de comunicación para redes de campo es ControlNet. Este protocolo se utiliza para la comunicación de chasis remotos y expansiones de rack.

-El protocolo de comunicación para PLC y Sistema supervisorio es Ethernet/IP 
-El driver de comunicación para comunicar PLC y Sistema supervisorio es OPC

Con estos criterios de comunicación, el personal de la Superintendencia de control de procesos ha obtenido mejores resultados, que siguiendo los protocolos PROFIBUS o DataHighWay.

La marca de PLC que mejor se adapta a estos criterios es Allen Bradley y las gamas de ControlLogix y Flex I/O ya que las otras marcas no ofrecen los protocolos de comunicación establecidos. La gama de Flex I/O es la gama de bajo costo de Allen Bradley. Es un autómata modular y ofrece todas las funciones de la gama de ControlLogix, con la limitación de que solo admite configuraciones de chasis de hasta 8 módulos. Como máximo, en esta gama solo se pueden manipular 256 señales digitales con un solo procesador y la aplicación para este proyecto demanda más de 500 señales digitales, por tanto la gama indicada para la aplicación es la gama ControlLogix.

\section{V.CONCLUSIONES}

-La revisión de los manuales técnicos del autómata S5-115U de Siemens, permitió la comprensión de esta plataforma de siemens, a nivel de hardware, software, configuración y programación. Toda ésta información fue necesaria para comprender el sistema de control que se desea sustituir y generar las bases y los criterios de diseño en el nuevo sistema de control, así como también permitió elaborar un levantamiento de la información del sistema instalado.

-Se logró seleccionar y configurar módulos E/S, procesadores y tarjetas de comunicación adecuados para el nuevo autómata a partir del levantamiento de la información del sistema instalado y de los criterios de diseño planteados.

-Se obtuvo una arquitectura de hardware nueva con garantías de abastecimiento de repuestos y reemplazos por parte del fabricante por diez años o más.

\section{REFERENCIAS}

[1] F. Bogadi. "Optimización del sistema supervisorio de la planta de aire comprimido de la empresa CVG CARBONORCA”, Instituto universitario Santiago Mariño, Venezuela, 2005.

[2] R. Coronado. "Diseño del sistema de control y supervisión para el sistema de manejo de ánodos cocidos (Cadena U) de CVG CARBONORCA”, Instituto universitario Santiago Mariño, Venezuela, 2005

[3] A. Pérez. "Modernización del sistema de control y supervisión local del manejo de ánodos verdes en la nave de cocción 1.2 de C.V.G. CARBONORCA”, UNEXPO-Vice Rectorado Puerto Ordaz, Ciudad Guayana, 2006.

[4] I. Mora. "Implementación del driver de comunicación entre el PLC S5-115U SIEMENS y el Sistema de supervisión iFIX Dynamics para supervisar el sistema de depuración de gases LURGI 1.2 de la empresa C.V.G CARBONORCA”, Instituto universitario Santiago Mariño, Venezuela, 2006.

[5] J. Rodríguez. "Diseño el sistema de control para la bomba de alquitrán líquido del sistema de dosificación de la planta de molienda y compactación de C.V.G. Carbonorca”, UNEXPO-Vice Rectorado Puerto Ordaz, Estado Bolívar, 2010.

[6] F. Osorio. "actualización del sistema de control del recuperador indoor SR71-1 en el área de manejo de materiales en C.V.G Bauxilum”, UNEXPO-Vice Rectorado Puerto Ordaz, Ciudad Guayana, 2010

[7] C. Bermúdez y F. Suárez. "Modernización de los automatas programables de las plantas depuradoras de gas, LURGI 1.1 y LURGI 1.2 de C.V.G. CARBONORCA”. UNEXPO Puerto Ordaz. Venezuela, 2011 


\section{RESUMEN CURRICULAR}

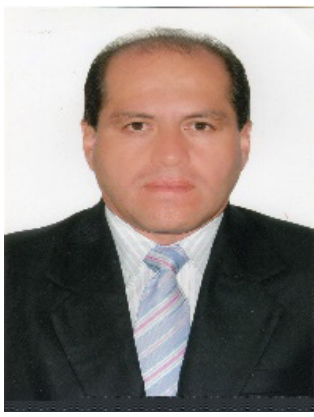

Ceballos Bejarano Edison Wernher, Ingeniero Electrónico, Magíster en Ingeniería Electrónica con Mención en Automatización e Instrumentación. Docente asociado en el programa de estudios de Ingeniería Electrónica, Docente investigador de la Universidad Nacional de San Agustín, Arequipa, Perú.

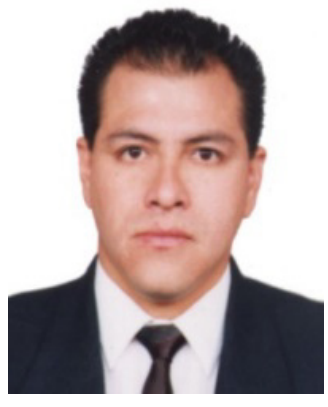

Ceballos Bejarano, Ferdinand Eddington, Licenciado en Matemática, Magíster en Finanzas y Administración de Negocios, Doctor en Ciencias Empresariales. Docente auxiliar en el programa de estudios de Matemáticas, Administración y Finanzas; miembro del Instituto de Investigaciones Sociales de la Universidad Nacional de San Agustín, Arequipa, Perú 